\section{Ansiedade na gestação, prematuridade e baixo peso ao nascer: uma revisão sistemática da literatura}

\author{
Anxiety during pregnancy, prematurity, and low \\ birth weight: a systematic literature review
}

\section{Abstract}

The purpose of this systematic literature review was to examine publications that had investigated the effect of anxiety on prematurity and/or low birth weight. The PubMed, BVS, CINAHL, and HEALTHSTAR databases, published from 1966 to 2006, were tracked using the following key words: "anxiety", "pregnancy", "low birth weight", and "prematurity". Thirteen studies were found: 11 cohorts, 1 cross-sectional, and 1 case-control. Most studies (7/13) were conducted in the United States. The most reliable results came from four studies, whose strengths were: exclusion of adolescents and/or women older than 34 years, studies that analyzed anxiety during the second and/or third trimester of pregnancy, used validated scales to measure anxiety, kept loss-to-follow-up rates below 30\%, and applied adequate control of confounders. Anxiety during pregnancy was associated with prematurity and/or low birth weight in eight studies. Odds ratios and relative risks varied from 1.08 to 2.31. Carefully designed and well-conducted studies are still needed to clarify the relationship between anxiety during pregnancy, prematurity, and low birth weight considering that the accumulated evidence remains controversial.

Anxiety; Pregnancy; Premature Infant; Low Birth Weight Infant; Review [Publication Type]
Daniele Marano Rocha Araújo 1

Natália de Lima Pereira 1

Gilberto Kac 1

\section{Introdução}

O peso ao nascer, obtido na primeira hora após o nascimento, reflete as condições nutricionais do recém-nascido e da gestante, sendo considerado indicador apropriado de saúde individual. Esse indicador influencia o crescimento e o desenvolvimento da criança e, em longo prazo, repercute nas condições de saúde do adulto 1 .

O baixo peso ao nascer é definido pela Organização Mundial da Saúde (OMS) como todo nascido vivo com peso ao nascer inferior a 2.500 gramas, e a prematuridade é classicamente definida como o nascimento que ocorre antes da 37a semana de gestação 2 .

A prematuridade e o baixo peso ao nascer são fatores determinantes da mortalidade neonatal, de infecções, de maiores taxas de hospitalização, maior propensão ao retardo de crescimento, déficit neuropsicológico pós-natal e baixo desempenho escolar 3,4,5,6.

Embora tenha havido um significativo avanço na medicina e tecnologia, a prevalência de nascimentos prematuros em países desenvolvidos como a França ainda é de $6 \%$, enquanto nos Estados Unidos é de $11 \%$ 7,8. Já nos países em desenvolvimento, como o Brasil, a prevalência de prematuros é de aproximadamente $7 \%$. A prevalência de baixo peso ao nascer é de $9,2 \%$ no Brasil 3, 3,3\% na Dinamarca, 30\% na Índia e 7\% nos Estados Unidos 9. 
Estudos epidemiológicos demonstram que existem inúmeros fatores responsáveis pelo desencadeamento desses processos, como por exemplo, as condições sócio-econômicas precárias, o peso da mãe antes e durante a gestação, a etnia/raça, a estatura, a idade, a escolaridade materna, os nascimentos múltiplos, a paridade, a história obstétrica anterior, os cuidados prénatais, a morbidade materna durante a gravidez, comportamentos de risco como o consumo de bebidas alcoólicas, a ingestão de café e o hábito de fumar 1,9,10,11,12,13,14.

Mais recentemente, alguns estudos vêm apontando a ansiedade como possível fator determinante tanto da prematuridade quanto do baixo peso ao nascer 7,9,11,14,15,16. A presença da ansiedade pode afetar negativamente a gestação por ser considerada mediadora de mudanças endócrinas como também de determinados comportamentos de risco, tais como o hábito de fumar, atraso e/ou inadequado acesso ao prénatal, alimentação e ganho de peso gestacional inadequado 17,18 .

Os quadros de ansiedade são muito freqüentes e deletérios à mulher em idade reprodutiva. Estima-se que $20 \%$ das mulheres apresentam sintomas de ansiedade durante a gravidez. Observa-se em estudos epidemiológicos que a ansiedade na gravidez é subdiagnosticada por vergonha das mulheres em apresentar suas queixas e por este sintoma ser muitas vezes confundido com problemas orgânicos 19 .

Dessa forma, esta revisão tem como objetivo examinar estudos que investigaram o efeito da ansiedade durante a gestação e o maior risco de prematuridade e/ou baixo peso ao nascer. A ênfase dada na discussão dos resultados é sobre questões metodológicas referentes à confiabilidade das escalas que medem ansiedade e ao controle insuficiente dos fatores de confusão, que podem distorcer os resultados das associações.

\section{Métodos}

O método da revisão sistemática da literatura foi utilizado. Esse método consiste na revisão retrospectiva de artigos científicos, neste caso sobre a associação entre ansiedade na gestação e os seguintes desfechos: prematuridade e baixo peso ao nascer.

Os artigos foram identificados por meio de busca na base de dados MEDLINE versão PubMed (http://www.pubmed.gov), Biblioteca Virtual em Saúde - BVS (http://www.bireme.br), CINAHL e HEALTHSTAR, referentes aos anos de 1966 a 2006. Os seguintes descritores foram utilizados na busca: anxiety, pregnancy, low birth weight, prematurity OR anxiety, pregnancy, prematurity OR anxiety, pregnancy, low birth weight OR anxiety, prematurity OR anxiety, low birth weight OR anxiety, low birth weight, prematurity. Outra estratégia foi a busca manual em listas de referências dos artigos identificados e selecionados.

O tipo de desenho do estudo (coorte, casocontrole ou transversal) e o idioma (português, inglês ou espanhol) foram os dois critérios utilizados na seleção de artigos para a revisão. Os artigos selecionados foram comparados em relação aos seguintes eixos: país de origem, idade média dos participantes, tamanho da amostra, tipo de desenho, taxa de perdas de seguimento, número de vezes que a exposição foi aferida, fatores de confusão controlados na análise e os principais resultados observados. Atenção especial foi dada à validade das escalas utilizadas para medir ansiedade, utilizando-se para este fim informações referentes à confiabilidade.

O termo confiabilidade é utilizado para se referir à reprodutibilidade de uma medida, ou seja, o grau de concordância entre múltiplas medidas de um mesmo objeto ${ }^{6}$. $\mathrm{O}$ aspecto que foi analisado em todos os estudos selecionados foi o grau de consistência interna das escalas utilizadas para mensurar a ansiedade na gestação. A consistência interna das escalas foi verificada pelo alpha de Cronbach. Esse parâmetro reflete o grau de co-variância dos itens entre si. Valores próximos de 1 indicam boa consistência interna. Para pesquisas exploratórias, aceitam-se valores acima de 0,6 .

Os estudos selecionados tiveram como desfecho a prematuridade e/ou o baixo peso ao nascer $7,9,11,12,14,15,16,17,20,21,22,23,24$.

\section{Resultados}

Segundo a estratégia estabelecida, a busca bibliográfica resultou em 425 artigos. Entretanto, após a implementação dos critérios de exclusão foram selecionados apenas dez artigos, sendo que três foram obtidos através da busca manual das referências selecionadas, totalizando 13 artigos.

Dos 13 estudos, 7 foram realizados nos Estados Unidos, 1 no Brasil, 2 na Noruega, 1 no Canadá, 1 na Dinamarca e 1 na Inglaterra. Em relação à faixa etária, sete estudos incluíram gestantes com menos de 19 e acima de 34 anos, um estudo informou apenas a idade média, dois estudos informaram a média e a idade mínima e um estudo não informou a idade das gestantes avaliadas (Tabela 1).

Dos 13 estudos selecionados, 11 utilizaram o desenho de coorte, 1 de caso-controle e 1 
Ano de publicação, origem, tamanho amostral e idade das gestantes dos estudos selecionados sobre fatores associados entre ansiedade na gestação, prematuridade e baixo peso ao nascer, 1966-2006.

\begin{tabular}{|c|c|c|c|c|}
\hline Autores & Ano de publicação & País & Amostra (n) & Idade (anos) \\
\hline Burstein et al. 20 & 1973 & Canadá & 61 & $17-42$ \\
\hline Pagel et al. 22 & 1990 & Estados Unidos & 100 & $21-31$ \\
\hline Wadhwa et al. 16 & 1993 & Estados Unidos & 90 & > 18; 24,7 (média) \\
\hline Zimmer-Gembeck \& Helfand 9 & 1996 & Estados Unidos & 3.073 & 19-34; 24,7 (média) \\
\hline Cooper \& Rachel 14 & 1996 & Estados Unidos & 2.593 & 22,6 (média) \\
\hline Bhagwanani et al. 11 & 1997 & Estados Unidos & 88 & $18-34$ \\
\hline Grimstad et al. 17 & 1999 & Noruega & 85 casos; 92 controles & $25-34$ \\
\hline Teixeira et al. 24 & 1999 & Inglaterra & 100 & $\mathrm{NI}$ \\
\hline Kelly et al. 21 & 2002 & Estados Unidos & 521.490 & $20-35$ \\
\hline Rondo et al. 15 & 2003 & Brasil & 865 & $19-34$ \\
\hline Mancuso et al. 7 & 2004 & Estados Unidos & 282 & $>18 ; 27,5$ (média) \\
\hline Berle et al. 12 & 2005 & Noruega & 680 & $20-49$ \\
\hline Neggers et al. 23 & 2006 & Dinamarca & 3.149 & $21-31$ \\
\hline
\end{tabular}

NI: não informado.

transversal. Em relação ao momento em que a exposição foi aferida, um dos estudos iniciou a avaliação das gestantes no primeiro trimestre, quatro no segundo, cinco somente no terceiro trimestre de gestação e três estudos não relataram o momento da gestação em que foi feita a aferição da ansiedade. Informações sobre as perdas de seguimento foram obtidas em 9 dos 13 estudos. As perdas variaram entre $10,3 \%$ no estudo de Burstein et al. 20 a 36,9\% no estudo de Mancuso et al. 7 (Tabela 2).

Entre os estudos selecionados, três demonstraram associação significativa entre ansiedade, prematuridade e baixo peso ao nascer, três apresentaram associação significativa entre ansiedade e prematuridade, dois entre ansiedade e baixo peso ao nascer, enquanto cinco estudos não evidenciaram associação entre ansiedade e os desfechos selecionados. Os valores de odds ratio e de risco relativo variaram de 1,08 a 2,31 (Tabela 3).

Em relação às escalas utilizadas para medir ansiedade, cinco estudos utilizaram o Inventário de Ansiedade Traço-Estado (IDATE), enquanto oito fizeram uso das seguintes escalas: 5 ItemScale, Pregnancy-Specific Anxiety Scale, The Multiple Affect Adjective Checklist, 28-Item Psychosocial Scale, Hospital Anxiety and Depression Rating Scale (HADS) e Taylor Manifest Anxiety Scale. O estudo de Kelly et al. 21 utilizou a International Classification of Diseases (ICD-9-CM) para mensurar a ansiedade. Os valores do alpha de Cronbach variaram de 0,16 a 0,62 para ansiedade-estado do IDATE a 0,71 a 0,96 na 5 Item-Scale, sen- do que em cinco estudos não foram informados os valores do alpha de Cronbach (Tabela 4).

\section{Discussão}

Existem poucos estudos prospectivos que tenham descrito a associação entre ansiedade e prematuridade e/ou baixo peso ao nascer 7,9,11,14,15,16. Os estudos que investigaram a associação entre essas variáveis são contraditórios. Algumas inconsistências nessa associação podem ser atribuídas em parte à seleção inadequada do tipo de estudo, a não inclusão de grupo de comparação, inclusão de gestantes adolescentes e/ou gestantes acima de trinta anos, altas taxas de perda de seguimento, ausência de controle para variáveis de confusão, diferentes tempos de seguimento ou diferenças no momento no qual a exposição (ansiedade) foi aferida 12,16,22,25. Uma das razões do interesse em se estudar a associação entre ansiedade, prematuridade e baixo peso ao nascer decorre da relevante prevalência de ambos os desfechos e a necessidade de que novos potenciais fatores determinantes possam ser controlados, diminuindo assim, a freqüência desses eventos. As taxas observadas desses desfechos ainda são altas tanto em países em desenvolvimento como também em países desenvolvidos 21,26,27.

Um grande número de fatores atua antes e durante a gestação e exerce influência no peso ao nascer e na idade gestacional do neonato 9,15,16. A idade da mãe é uma variável de relevância em es- 
Características dos estudos selecionados sobre fatores associados entre ansiedade na gestação, prematuridade e baixo peso ao nascer, $1966-2006$.

\begin{tabular}{|c|c|c|c|}
\hline Autores & Tipo de desenho & $\begin{array}{l}\text { Perdas } \\
\text { (\%) }\end{array}$ & $\begin{array}{l}\text { Período em que a ansiedade foi observada } \\
\qquad \text { (semanas) }\end{array}$ \\
\hline Burstein et al. 20 & Coorte & 10,3 & 28a-36ạ \\
\hline Pagel et al. 22 & Coorte & $\mathrm{NI}$ & 21a-36ạ; 32 semanas (média) \\
\hline Wadhwa et al. 16 & Coorte & 11,7 & 28a-30ạ \\
\hline Zimmer-Gembeck \& Helfand 9 & Coorte & $\mathrm{NI}$ & 3o trimestre \\
\hline Cooper \& Rachel 14 & Coorte & 15,6 & 25ạ-29ạ \\
\hline Bhagwanani et al. 11 & Coorte & 31,7 & $8 \underline{a}, 20 \underline{a}, 21 \underline{a}-28 \underline{a}$ \\
\hline Grimstad et al. 17 & Caso-controle & 19,2 & $\mathrm{NI}$ \\
\hline Teixeira et al. 24 & Coorte & 15,9 & 3o trimestre; 32 semanas (média) \\
\hline Kelly et al. 21 & Coorte retrospectiva & 0,0 & $\mathrm{NI}$ \\
\hline Rondo et al. 15 & Coorte & 26,8 & $<$ 16ạ, 20ạ-26ạ, 30a-36 \\
\hline Mancuso et al. 7 & Coorte & 36,9 & 18a-20ạ, 28a-30ạ, 35ạ-36ạ \\
\hline Berle et al. 12 & Transversal & $\mathrm{NI}$ & Ao longo da gestação \\
\hline Neggers et al. 23 & Coorte & 22,5 & 22a-23ạ \\
\hline
\end{tabular}

NI: não informado.

tudos demográficos e epidemiológicos. Entre os estudos analisados nesta revisão alguns incluíram gestantes adolescentes ( $<18$ anos) e/ou mulheres com idade superior a trinta anos. Ximenes \& Oliveira 28 apontaram que o interesse pelos efeitos da idade sobre a fertilidade, a saúde do recém-nascido e da mãe ocorre devido ao aumento do número de gestantes adolescentes e mulheres que engravidam pela primeira vez depois dos trinta anos. Inúmeros estudos têm demonstrado que adolescentes e mulheres acima de 35 anos possuem maiores riscos de morbidade e mortalidade durante a gravidez, como também maiores chances de gerarem filhos prematuros e de baixo peso $11,15,26,28,29,30$.

Os estudos selecionados apresentaram diferentes momentos nos quais a exposição (ansiedade) e os desfechos (prematuridade e baixo peso ao nascer) foram mensurados, o que pode justificar, em parte, as diferenças nos resultados encontrados 11 . Os estudos conduzidos por Rondo et al. ${ }^{15}$ e por Mancuso et al. ${ }^{7}$ avaliaram a ansiedade em três momentos durante a gestação, sendo a primeira vez sempre depois do segundo trimestre. Os demais estudos realizaram apenas uma aferição, que ocorreu em diferentes momentos da gestação. Segundo Andersson et al. 31, pode haver questionamento quando se realiza apenas uma entrevista para mensurar sintomas psiquiátricos como a ansiedade durante a gravidez, pelo fato de tais sintomas não terem sido modificados, serem transitórios ou desenvolvidos após a entrevista.
As perdas de seguimento podem ocasionar diminuição na validade dos resultados. Observou-se perda superior a $30 \%$ em dois estudos 7,11. As estimativas de associação podem estar comprometidas pelo fato de alguns estudos não terem quantificado as perdas de seguimento, como também por não terem realizado o controle de alguns importantes fatores de confusão 9,12,14 .

Em relação ao estado sócio-econômico, o estudo conduzido por Wadhwa et al. 16 foi composto por mulheres de classe média ou superior, já os estudos de Zimmer-Gembeck \& Helfand 9 e o de Neggers et al. 23 foram compostos predominantemente por mulheres de baixa renda. Nos demais estudos não houve homogeneidade quanto às características sócio-econômicas. A desvantagem sócio-econômica é considerada um fator etiológico indireto tanto para a prematuridade quanto para o baixo peso ao nascer, pois pode mediar exposições psicológicas, comportamentais e ambientais envolvidas na redução do crescimento fetal 12 .

O estudo conduzido por Mancuso et al. 7 investigou o efeito de fatores endócrinos e psicológicos na prematuridade. Evidências sugerem que a ansiedade aumenta a secreção do hormônio corticotrópico (CRH). Sabe-se que esse hormônio interage com as prostaglandinas e oxitocinas, mediadoras da contração uterina. Esse estudo controlou riscos médicos e fatores sócio-econômicos, porém, as pacientes eram etnicamente heterogêneas. O entendimento dos fatores endócrinos ainda é incipiente, no entanto este tra- 
Fatores controlados na análise e tipo de associação encontrada entre ansiedade, prematuridade e baixo peso ao nascer, $1966-2006$.

\begin{tabular}{|c|c|c|c|c|}
\hline Autores & Desfechos & $\begin{array}{l}\text { Fatores de confusão } \\
\text { controlados na análise }\end{array}$ & Resultados & Estimadores \\
\hline Burstein et al. 20 & Baixo peso ao nascer & $\mathrm{NI}$ & $\begin{array}{l}\text { A ansiedade não foi correlacionada } \\
\text { ao baixo peso ao nascer utilizando } \\
\text { o instrumento Taylor Manifest Anxiety } \\
\text { Scale }(r=-0,097 ; p=0,228) \text { e } \\
\text { Pregnancy-Specific Anxiety Scale } \\
(r=-0,104 ; p=0,213)\end{array}$ & $\begin{array}{c}\text { r (coeficiente } \\
\text { de correlação) }\end{array}$ \\
\hline Pagel et al. 22 & Prematuridade & $\begin{array}{l}\text { Influência dos fatores biomédicos } \\
\text { (diabetes, doenças cardíacas, } \\
\text { hipertensão, problemas tireoi- } \\
\text { dianos, infecção urinária, asma, } \\
\text { fadiga, náusea, pré-eclâmpsia) }\end{array}$ & $\begin{array}{l}\text { A ansiedade foi correlacionada à } \\
\text { prematuridade }(r=0,24)\end{array}$ & $\begin{array}{l}r \text { (coeficiente } \\
\text { de correlação) }\end{array}$ \\
\hline Wadhawa et al. 16 & $\begin{array}{l}\text { Baixo peso ao nascer } \\
\text { e prematuridade }\end{array}$ & $\begin{array}{l}\text { Riscos médicos (diabetes, } \\
\text { eclâmpsia, morte fetal, herpes, } \\
\text { hipertensão, doenças } \\
\text { coronarianas, ruptura prematura } \\
\text { da membrana e retardo do } \\
\text { crescimento intra-uterino) }\end{array}$ & $\begin{array}{l}\text { A análise multivariada demonstrou que } \\
\text { a ansiedade na gestação não foi } \\
\text { associada ao baixo peso ao nascer } \\
(p=0,11) \text {, porém demonstrou } \\
\text { associação significativa com a } \\
\text { prematuridade }(\beta=-0,42 ; p<0,05)\end{array}$ & Coeficiente $\beta$ \\
\hline $\begin{array}{l}\text { Zimmer-Gembeck } \\
\& \text { Helfand } 9\end{array}$ & Baixo peso ao nascer & $\begin{array}{l}\text { Riscos médicos (história anterior } \\
\text { de baixo peso ao nascer, } \\
\text { hipertensão e doença renal) }\end{array}$ & $\begin{array}{l}\text { Ansiedade, depressão e hostilidade } \\
\text { foram associadas ao risco aumentado } \\
\text { para o baixo peso ao nascer (OR }=1,65 \text {; } \\
\text { IC95\%: } 1,12-2,42 ; p=0,012)\end{array}$ & OR \\
\hline Cooper \& Rachel. 14 & $\begin{array}{l}\text { Baixo peso ao nascer } \\
\text { e prematuridade }\end{array}$ & $\begin{array}{l}\text { Raça, idade materna, estado } \\
\text { marital, escolaridade, tabagismo, } \\
\text { álcool e drogas }\end{array}$ & $\begin{array}{l}\text { Entre as variáveis psicológicas } \\
\text { avaliadas, apenas o estresse foi } \\
\text { associado ao baixo peso ao nascer } \\
(\mathrm{OR}=1,8 ; p=0,02) \text { e à prematuridade } \\
(\mathrm{OR}=1,16 ; p=0,003)\end{array}$ & OR \\
\hline Bhagwanani et al. 11 & $\begin{array}{l}\text { Baixo peso ao nascer } \\
\text { e prematuridade }\end{array}$ & $\begin{array}{l}\text { Doenças crônicas, idade } \\
\text { gestacional e complicações } \\
\text { no pré-parto }\end{array}$ & $\begin{array}{l}\text { Observou-se diferença significativa na média } \\
\text { do escore de relação entre ansiedade-traço } \\
(p=0,052 ; \text { média }=34,8) \text { e ansiedade-estado } \\
(p=0,004 ; \text { média }=35,1) \text { em crianças com } \\
\text { e sem baixo peso ao nascer. Também se } \\
\text { observou diferença significativa no escore } \\
\text { entre ansiedade-traço ( } p=0,019 ; \\
\text { média }=32,8) \text { e ansiedade-estado } \\
(p=0,017 ; \text { média }=31,5) \text { em crianças } \\
\text { com e sem prematuridade }\end{array}$ & Média \\
\hline Grimstad et al. 17 & Baixo peso ao nascer & $\mathrm{NI}$ & $\begin{array}{l}\text { Não houve relação entre ansiedade } \\
\text { e baixo peso ao nascer ( } p>0,05 \\
\text { média }=39,6 \text { - casos e } 39,7 \text { - controles) }\end{array}$ & Média \\
\hline Teixeira et al. 24 & Baixo peso ao nascer & $\begin{array}{l}\text { Doenças médicas (pré-eclâmpsia, } \\
\text { hemorragia, fumo, parto prematuro } \\
\text { anterior e bebês pequenos para a } \\
\text { idade gestacional, volume anormal } \\
\text { de líquido amniótico, gestações } \\
\text { múltiplas, velocidade anormal do } \\
\text { fluxo sanguíneo na artéria umbilical }\end{array}$ & $\begin{array}{l}\text { Correlação entre ansiedade-estado } \\
(r=0,31, p<0,02) \text {, ansiedade-traço } \\
(r=0,08 ; p<0,005) \text { e resistência da } \\
\text { artéria uterina acarretando baixo peso } \\
\text { ao nascer }\end{array}$ & $\begin{array}{l}r \text { (coeficiente } \\
\text { de correlação) }\end{array}$ \\
\hline Kelly et al. 21 & $\begin{array}{l}\text { Baixo peso ao nascer } \\
\text { e prematuridade }\end{array}$ & $\begin{array}{l}\text { Fatores demográficos, idade } \\
\text { materna, etnia, seguro de vida, } \\
\text { estado marital, educação, } \\
\text { paridade, diabetes e hipertensão }\end{array}$ & $\begin{array}{l}\text { Desordens psiquiátricas foram associadas } \\
\text { ao baixo peso ao nascer }(\mathrm{OR}=2,0 ; \mathrm{IC} 95 \% \text { : } \\
1,7-2,3) \text { e à prematuridade }(\mathrm{OR}=1,6 \text {; } \\
\text { IC } 95 \% \text { : } 1,4-1,9)\end{array}$ & OR \\
\hline
\end{tabular}

(continua) 


\begin{tabular}{|c|c|c|c|c|}
\hline Autores & Desfechos & $\begin{array}{l}\text { Fatores de confusão } \\
\text { controlados na análise }\end{array}$ & Resultados & Estimadores \\
\hline Rondo et al. 15 & $\begin{array}{l}\text { Baixo peso ao nascer } \\
\text { e prematuridade }\end{array}$ & $\begin{array}{l}\text { Idade, educação, estado marital, } \\
\text { renda, paridade, história prévia } \\
\text { de baixo peso ao nascer, peso } \\
\text { pré-gestacional e altura }\end{array}$ & $\begin{array}{l}\text { A análise de regressão logística demonstrou } \\
\text { que a ansiedade nas 20ạ-26a semanas } \\
\text { gestacionais foi associada ao baixo peso } \\
\text { ao nascer (RR }=1,97 ; \text { IC } 95 \%: 1,12-3,47 \\
\mathrm{p}=0,019) \text { e à prematuridade }(\mathrm{RR}=2,31 \\
\text { IC } 95 \%: 1,18-4,60 ; \mathrm{p}=0,015)\end{array}$ & RR \\
\hline Mancuso et al. 7 & Prematuridade & $\begin{array}{l}\text { Riscos médicos (histórico de } \\
\text { infertilidade, infecção vaginal e } \\
\text { no trato urinário, anemia, febre), } \\
\text { fumo, renda, escolaridade e } \\
\text { paridade }\end{array}$ & $\begin{array}{l}\text { A ansiedade no período pré-natal } \\
\text { (aferida entre as } 28 \text { a- } 30 \text { a semanas) foi } \\
\text { associada à prematuridade ( } \beta=-0,35 \\
p<0,05 \text { ), assim com aos altos níveis } \\
\text { de hormônio corticotrópico }\end{array}$ & Coeficiente $\beta$ \\
\hline Berle et al. 12 & $\begin{array}{l}\text { Baixo peso ao nascer } \\
\text { e prematuridade }\end{array}$ & $\begin{array}{l}\text { Variáveis demográficas, biológicas } \\
\text { e obstétricas }\end{array}$ & $\begin{array}{l}\text { Não houve associação entre ansiedade, } \\
\text { baixo peso ao nascer e prematuridade }\end{array}$ & $\mathrm{OR}$ * \\
\hline Neggers et al. 23 & Baixo peso ao nascer & $\begin{array}{l}\text { Raça, idade, índice de massa } \\
\text { corporal pré-gestacional, paridade, } \\
\text { escolaridade, álcool, tabagismo, } \\
\text { hipertensão, diabetes, parto } \\
\text { prematuro anterior }\end{array}$ & $\begin{array}{l}\text { Apenas a depressão foi associada ao } \\
\text { baixo peso ao nascer (OR =1,4; IC95\%: } \\
1,1-1,4 ; p=0,01)\end{array}$ & OR \\
\hline
\end{tabular}

NI: não informado; OR: odds ratio; IC95\%: intervalo de confiança de 95\%; RR: risco relativo.

* Não foi informado o valor de OR.

balho demonstrou associação da ansiedade presente entre as 28a e 30a semanas de gestação com o aumento do hormônio corticotrópico. Essa associação trouxe uma importante contribuição, já que a ansiedade durante o período gestacional pode ser modificada por meio de intervenções, que possivelmente acarretarão redução de nascimentos prematuros.

O estudo conduzido por Burstein et al. 20 mensurou a ansiedade-estado, como único constructo da ansiedade, durante o último trimestre da gestação, pois, segundo o autor, a ansiedade se torna mais manifesta próxima ao parto. Apesar do aumento do escore da ansiedade-estado nesse período, esse fator psicológico não foi associado ao baixo peso ao nascer.

Entre os estudos selecionados, apenas um relacionou a ansiedade durante a gestação, comportamentos maternos, raça, intervenção psicológica e o baixo peso ao nascer controlando para riscos médicos (história anterior de baixo peso ao nascer, hipertensão e doença renal) 9 . Também foi demonstrado que a assistência psicológica durante 45 minutos e/ou participar de mais de uma sessão nos serviços de psicologia foi responsável pela redução da incidência de baixo peso ao nascer naquela população.

Cinco estudos não demonstraram associação entre ansiedade, prematuridade e baixo peso ao nascer 12,14,17,20,23. No entanto, dois estudos demonstraram que a ansiedade pode afetar negativamente a gestação por ser considerada mediadora de determinados comportamentos de risco importantes para o baixo peso ao nascer, como o hábito de fumar e beber 14,17.

Entre os estudos selecionados, houve o uso de diversos tipos de escalas para medir ansiedade durante a gestação. Por isso, a comparação entre os mesmos também ficou dificultada, pois existem escalas que tentam cobrir todos os aspectos da ansiedade enquanto outras enfatizam um ou outro aspecto.

Keedwell \& Snaith 32 fizeram um levantamento sobre as escalas de ansiedade mais utilizadas nos últimos anos. Entre elas os autores destacaram as escalas de auto-avaliação (IDATE), Escala de Ansiedade de Zung, Taylor Manifest Anxiety Scale, Subescala de Ansiedade do Symptom Checklist e HADS. Segundo Oliveira \& Sisto 33, o IDATE é o mais comumente utilizado nas pesquisas epidemiológicas, corroborando com os dados desta revisão sistemática.

O IDATE foi inicialmente proposto como um instrumento de pesquisa com o objetivo de estudar a ansiedade na população adulta. Esse inventário é composto por duas escalas diferentes que têm como objetivo medir a ansiedade-traço e a ansiedade-estado 17,31,34,35,36. Segundo Spiel- 
Sintomas avaliados e confiabilidade das escalas utilizadas para medir ansiedade no período gestacional, $1966-2006$.

\begin{tabular}{|c|c|c|c|}
\hline Autores & Escala & Sintomas avaliados & Alpha de Cronbach \\
\hline \multirow[t]{2}{*}{ Burstein et al. 20} & Taylor Manifest Anxiety Scale & Ansiedade & $\mathrm{NI}$ \\
\hline & Pregnancy-Specific Anxiety Scale & Ansiedade & $0,65-0,72$ \\
\hline \multirow[t]{2}{*}{ Page et al. 22} & IDATE & Ansiedade-traço & Ansiedade-traço: 0,65-0,86 \\
\hline & & Ansiedade-estado & Ansiedade-estado: 0,16-0,62 \\
\hline Wadhwa et al. 16 & 5 Item-Scale & $\begin{array}{l}\text { Ansiedade em relação ao parto, } \\
\text { saúde do bebê, equipe de saúde }\end{array}$ & $0,71-0,96$ \\
\hline Zimmer-Gembeck \& Helfand 9 & The Multiple Affect Adjective Checklist & Ansiedade, depressão e hostilidade & $\mathrm{NI}$ \\
\hline Cooper \& Rachel 14 & 28-Item Psychosocial Scale & $\begin{array}{c}\text { Ansiedade-traço, auto-estima, autocontrole, } \\
\text { depressão, estresse subjetivo }\end{array}$ & $\mathrm{NI}$ \\
\hline \multirow[t]{2}{*}{ Bhagwanani et al. 11} & IDATE & Ansiedade-traço & Ansiedade-traço: 0,65-0,86 \\
\hline & & Ansiedade-estado & Ansiedade-estado: 0,16-0,62 \\
\hline \multirow[t]{2}{*}{ Grimstad et al. 17} & IDATE & Ansiedade-traço & Ansiedade-traço: 0,65-0,86 \\
\hline & & Ansiedade-estado & Ansiedade-estado: 0,16-0,62 \\
\hline \multirow[t]{2}{*}{ Teixeira et al. 24} & IDATE & Ansiedade-traço & Ansiedade-traço: 0,65-0,86 \\
\hline & & Ansiedade-estado & Ansiedade-estado: 0,16-0,62 \\
\hline Kelly et al. 21 & ICD-9-CM & Desordens psiquiátricas & $\mathrm{NI}$ \\
\hline \multirow[t]{2}{*}{ Rondo et al. 15} & IDATE & Ansiedade-traço & Ansiedade-traço: 0,65-0,86 \\
\hline & & Ansiedade-estado & Ansiedade-estado: 0,16-0,62 \\
\hline Mancuso et al. 7 & Pregnancy-Specific Anxiety Scale & Ansiedade & $0,65-0,72$ \\
\hline \multirow[t]{2}{*}{ Berle et al. 12} & HADS & Ansiedade (HADS-A) & HADS-A: 0,65-0,93 \\
\hline & & Depressão (HADS-D) & HADS-D: 0,80 \\
\hline Neggers et al. 23 & 28-Item Psychosocial Scale & $\begin{array}{c}\text { Ansiedade-traço, auto-estima, autocontrole, } \\
\text { depressão, estresse subjetivo }\end{array}$ & $\mathrm{NI}$ \\
\hline
\end{tabular}

NI: não informado; IDATE: Inventário de Ansiedade Traço-Estado; ICD-9-CM: International Classification of Diseases; HADS: Hospital Anxiety and Depression Rating Scale.

berguer et al. 36, a confiabilidade da escala de ansiedade-traço variou entre 0,65 e 0,86 , e da ansiedade-estado variou entre 0,16 e 0,62. A confiabilidade foi baixa para escala de ansiedade-estado devido à forte influência de fatores externos no momento da aferição. O IDATE teve correlação de 0,8 em relação à Taylor Manifest Anxiety Scale, 0,75 em relação à escala de ansiedade Personal Assessment Inventory (IPAT) e 0,52 em relação à Subescala de Ansiedade do Symptom Checklist.

A HADS foi desenvolvida para ser aplicada em pacientes de serviços não-psiquiátricos de hospital geral ou da atenção primária, a fim de identificar os sintomas de ansiedade e depressão. A escala possui 14 itens de múltipla escolha, sendo 7 para depressão (HADS-D) e 7 para ansiedade (HADS-A). Bjelland et al. 37 realizaram uma revisão que teve como objetivo avaliar a validade dessa escala, a qual demonstrou que a correlação entre as duas escalas variou entre 0,40 e 0,74 , e o alpha de Cronbach da HADS-A variou entre 0,68 e 0,93 e 0,67 e 0,90 na HADS-D. A sensibilidade e especificidade de ambas as escalas foi de 0,80 .
Apesar de a HADS ter sido desenvolvida para mensurar a ansiedade e depressão em pacientes hospitalares, demonstrou-se que esta escala tem as mesmas propriedades quando aplicada na população geral, sendo considerado um instrumento adequado para mensurar casos de desordens de ansiedade e depressão na população. Dessa forma, a HADS além de ter mensurado a confiabilidade, também aferiu estatisticamente a validade de critério por meio da sensibilidade $\mathrm{e}$ especificidade 37 .

A escala Abbreviated Scale for the Assessment of Psychosocial Status in Pregnancy é uma adaptação de cinco escalas previamente validadas que mensuravam ansiedade-traço, auto-estima, autocontrole, depressão e estresse subjetivo. Essas cinco escalas foram utilizadas previamente na avaliação de gestantes negras, multíparas e de baixa renda, que participaram de um estudo longitudinal que tinha como objetivo avaliar fatores de risco para retardo de crescimento intra-uterino. A ansiedade-traço, a auto-estima, o autocontrole e a depressão foram avaliados nas 
24a-26a semanas de gestação e o estresse subjetivo foi avaliado nas 30a-32a semanas de gestação ${ }^{38}$. Dessa forma, foi realizada uma análise prévia para criar um instrumento de avaliação mais fácil e com resultados similares às cinco escalas, que culminou na escala Likert-28 (28-Item Scale), utilizada no estudo de Cooper \& Rachel 14 e de Neggers et al. 23. A validade dessa escala foi considerada adequada para avaliar a associação entre características psicossociais e desfechos perinatais 38 .

A escala Pregnancy-Specific Anxiety Scale, utilizada no estudo de Mancuso et al. 7, foi desenvolvida pelos próprios investigadores para avaliar a ansiedade na gravidez. A análise da consistência interna dessa escala resultou em um alpha de Cronbach de 0,72 na avaliação feita entre as 18 a e 20a semanas e de 0,65 na avaliação entres as 28a e 30a semanas de gestação.

No estudo de Wadhwa et al. 16, a escala utilizada para mensurar a ansiedade na gestação foi a 5 Item-Scale, que apresentou consistência interna variando entre 0,71 e 0,96. Esse instrumento apresentou como objetivo avaliar os sentimentos maternos relacionados à saúde do bebê, parto, nascimento e confiança na equipe de saúde. $\mathrm{O}$ valor do alpha de Cronbach das escalas variou de 0,16 a 0,96 . Dessa forma, com exceção da escala ansiedade-estado, advinda do IDATE, onde o alpha variou de 0,16 a 0,62, podemos indicar que as escalas apresentam propriedades psicométricas adequadas para avaliação de gestantes.

O estudo conduzido por Burstein et al. 20 utilizou a Taylor Manifest Anxiety Scale e a Pregnancy Anxiety Scale. As duas escalas possuem duas únicas opções de resposta: verdadeiro ou falso, com 50 itens e 25 itens, respectivamente. A Taylor Manifest Anxiety Scale apresenta constructos semelhantes e tem como ênfase os aspectos somáticos da ansiedade 33

A limitação desta revisão foi ter pesquisado artigos escritos apenas em inglês, espanhol e português.

A relação entre a ansiedade no período gestacional, prematuridade e baixo peso ao nascer ainda tem recebido pequena atenção da comunidade científica, pois os estudos ainda dão maior importância a fatores que já foram estudados exaustivamente, tais como o fumo, consumo de bebidas alcoólicas, peso materno, assistência pré-natal, entre outros.

Dessa forma, a realização de estudos cuidadosamente desenhados com recrutamento de gestantes com características similares e com a utilização de escalas validadas, pode contribuir para a produção de novas evidências acerca da relação entre ansiedade, prematuridade e baixo peso ao nascer. Essas evidências podem ser importantes para subsidiar o profissional de saúde na aplicação preventiva de uma abordagem adequada ao enfrentamento do problema. 


\section{Resumo}

O objetivo desta revisão é examinar publicações que investigaram o efeito da ansiedade no maior risco de prematuridade elou baixo peso ao nascer. Os bancos de dados MEDLINE versão PubMed, BVS, CINAHL e HEALTHSTAR, referentes aos anos de 1966 a 2006, foram rastreados usando-se a combinação dos seguintes descritores: anxiety, pregnancy, low birth weight $\mathrm{e}$ prematurity. Foram localizados 13 estudos: 11 coortes, 1 transversal e 1 caso-controle. A maioria (7/13) dos estudos foi realizado nos Estados Unidos. Quatro estudos foram considerados de excelente qualidade, pois excluíram adolescentes elou mulheres acima de 34 anos, aferiram a ansiedade a partir do primeiro elou segundo trimestre de gestação, utilizaram escalas validadas para medir ansiedade, apresentaram perdas de seguimento inferiores a 30\% e controlaram os mais importantes fatores de confusão. A ansiedade na gestação foi associada à prematuridade elou ao baixo peso ao nascer em oito estudos. Os valores de razão de chance e risco relativo variaram de 1,08 a 2,31. São necessários estudos cuidadosamente desenhados para esclarecer a relação entre ansiedade na gestação, prematuridade e baixo peso ao nascer, já que as evidências observadas ainda são contraditórias.

Ansiedade; Gravidez; Prematuro; Recém-Nascido de Baixo Peso; Revisão [Tipo de Publicação]

\section{Referências}

1. Motta ME, Silva GA, Araújo OC, Lira PI, Lima MC. $\mathrm{O}$ peso ao nascer influencia o estado nutricional ao final do primeiro ano de vida? J Pediatr (Rio de J) 2005; 81:377-82.

2. WHO: recommended definitions, terminology and format for statistical tables related to the perinatal period and use of a new certificate for cause of perinatal deaths. Modifications recommended by FIGO as amended October 14, 1976. Acta Obstet Gynecol Scand 1977; 56:247-53.

3. Guimarães AAG, Velásquez-Meléndez G. Determinantes do baixo peso ao nascer a partir do sistema de informação sobre nascidos vivos em Itaúna, Minas Gerais. Rev Bras Saúde Matern Infant 2002; 2:283-90.

4. Mattison DR, Damus K, Fiore E, Petrini J, Alter C. Preterm delivery: a public health perspective. Paediatric Perinatal Epidemiol 2001; 15:1-7.

5. McCormick MC, Brooks-Gunn J, Shorter T. Factors associated with smoking in low income pregnant women: relationship to birth weight, stressful life events, social support, health behaviors and mental distress. J Clin Epidemiol 1990; 43:441-8.

\section{Colaboradores}

D. M. R. Araújo fez a revisão sistemática da literatura e participou de todas as etapas de elaboração do artigo. N. L. Pereira colaborou na revisão sistemática da literatura e participou de todas as etapas de elaboração do artigo. G. Kac participou de todas as etapas de elaboração do artigo.
6. Menezes AMR, Barros FC, Victora CG, Tomasi E, Halpern R, Oliveira ALB. Fatores de risco para a mortalidade perinatal em Pelotas, RS, 1993. Rev Saúde Pública 1998; 32:209-16.

7. Mancuso RA, Schetter CD, Rini CM, Roesch SC, Hobel CJ. Maternal prenatal anxiety and corticotropin-releasing hormone associated with timing of delivery. Psychosom Med 2004; 66:762-9.

8. Medeiros A, Gouveia N. Relação entre baixo peso ao nascer e a poluição do ar no Município de São Paulo. Rev Saúde Pública 2005; 39:965-72.

9. Zimmer-Gembeck MJ, Helfand M. Low birthweight in a public prenatal care program: behavioral and psychosocial risk factors and psychosocial intervention. Soc Sci Med 1996; 43:187-97.

10. Bicalho GG, Barros Filho AA. Peso ao nascer e influência do consumo de cafeína. Rev Saúde Pública 2002; 36:180-7.

11. Baghwanani SG, Seagraves K, Dierker LJ, Lax M. Relationship between prenatal anxiety and perinatal outcome in nulliparous women: a prospective study. J Natl Med Assoc 1997; 89:93-8. 
12. Berle JO, Mykletum A, Daltveit AK, Rasmussen S, Holtsten F, Dahl AA. Neonatal outcomes in offspring of women with anxiety and depression during pregnancy. A linkage study from the nord-trondelag health study (HUNT) and medical birth registry of Norway. Arch Womens Ment Health 2005; 8:181-9.

13. Costa CE, Gotlieb SLD. Estudo epidemiológico do peso ao nascer a partir da declaração de nascido vivo. Rev Saúde Pública 1998; 32:328-34.

14. Cooper MSN, Rachel L. The preterm prediction study: maternal stress is associated with spontaneous preterm birth at less than thirty five weeks gestation. Am J Obstet Gynecol 1996; 175:1286-92.

15. Rondo PHC, Ferreira, RF, Nogueira F, Ribeiro MCN, Lobert H, Artes R. Maternal psychological stress and distress as predictors of low birth weight, prematurity and intrauterine growth retardation. Eur J Clin Nutr 2003; 57:266-72.

16. Wadhwa PD, Sandman CA, Porto M, Shetter-Dunkel C, Garite TJ. The association between prenatal stress and infant birth weight and gestational age at birth: a prospective investigation. Am J Obstet Gynecol 1993; 169:858-65.

17. Grimstad H, Scheir B, Backe B, Jacobsen G. Anxiety, physical abuse, and low birth weight. Scand J Public Health 1999; 27:296-300.

18. Orr S, James SA, Miller CA, Barakat B. Psychosocial stressors and low birth weight in an urban population. Am J Prev Med 1996; 12:459-66.

19. Gouveia VV, Chaves SSS, Oliveira ICP, Dias M, Gouveia $\mathrm{R}$, Andrade $\mathrm{P}$. The use of the GHQ-12 in a general population: a study of its construct validity. Psicol Teor Pesqui 2003; 19:241-8.

20. Burstein I, Kinch R, Stern L. Anxiety, pregnancy, labor, and the neonate. Am J Obstet Gynecol 1974; 118:195-9.

21. Kelly RH, Russo J, Holt V, Danielsen BH, Zatzick DF, Walker E, et al. Psychiatric and substance use disorders as risk factors for low birth weight and preterm delivery. Obstet Gynecol 2002; 100:297-304.

22. Pagel MD, Smilxtein G, Regen H, Montano D. Psychosocial influences on new born outcomes: a controlled prospective study. Soc Sci Med 1990; 30:597-604.

23. Neggers Y, Goldenberg R, Cliver S, Hauth J. The relationship between psychosocial profile, health practices, and pregnancy outcomes. Acta Obstet Gynecol 2006; 85:277-85.
24. Teixeira J, Fisk N, Glover V. Association between maternal anxiety in pregnancy and increased uterine artery resistance index: cohort based study. BMJ 1999; 16:153-7.

25. Standley K, Soule B, Copans SA. Dimensions of prenatal anxiety and their influence on pregnancy outcome. Am J Obstet Gynecol 1979; 135:22-6.

26. Branun AM, Schoendorf KC. Changing patterns of low birth weight in the United States. Paediatr Perinatal Epidemiol 2002; 16:8-15.

27. Horta BL, Barros FC, Halpern R, Victora CG. Baixo peso ao nascer em duas coortes de base populacional no Sul do Brasil. Cad Saúde Pública 1996; 12 Suppl 1:S27-31.

28. Ximenes FMA, Oliveira MCR. A influência da idade materna sobre as condições perinatais. Rev Bras Promoção Saúde 2004; 17:56-60.

29. Brown HL, Fan YD, Gonsoulin WJ. Obstetric complications in young teenagers. South Med J 1991; 84:46-8.

30. Taquete SR. Sexo e gravidez na adolescência: estudo de antecedentes biopsicossociais. J Pediatr (Rio de J) 1992; 68:135-9.

31. Andersson L, Sundstrom-Poromaa I, Bixo M, Wulff $\mathrm{M}$, Bondestam K, Astrom M. Point prevalence of psychiatric disorders during the second trimester of pregnancy: A population- based study. Am J Obstet Gynecol 2003; 189:148-54.

32. Keedweel P, Sanith RP. What do anxiety scales measure? Acta Psychiatr Scand 1996; 93:177-80.

33. Oliveira JCS, Sisto FF. Construção de uma escala de ansiedade para pacientes de ambulatório: um estudo exploratório. Psicol Teor Prát 2004; 6:45-57.

34. Andrade LHSG, Gorenstein C. Aspectos gerais das escalas de avaliação de ansiedade. Rev Psiquiatr Clín (São Paulo) 1998; 25:285-90.

35. La Rosa J. Ansiedade, sexo, nível sócio-econômico e ordem de nascimento. Psicol Reflex Crit 1998; 11:59-70.

36. Spielberger CD, Gorusch RL, Lushene RE. Statetrait anxiety inventory. Palo Alto: Consulting Psychologists Press; 1970

37. Bjelland I, Dahl AA, Haug TT, Neckelmann D. The validity of the Hospital Anxiety and Depression Scale. An updated literature review. J Psychosom Res 2002; 52:69-77.

38. Goldenberg RL, Hickey CA, Cliver SP, Gotlieb S, Woolley TW, Hoffman HJ. Abbreviated scale for the assessment of psychosocial status in pregnancy: development and evaluation. Acta Obstet Gynecol Scand Suppl 1997; 165:19-29.

Recebido em 02/Jun/2006

Versão final reapresentada em 20/Dez/2006

Aprovado em 11/Jan/2007 